\title{
GRUPOS DE PESQUISA EM EDUCAÇÃO EM ENFERMAGEM: CARACTERIZAÇÃO DE TRÊS REGIÕES BRASILEIRAS
}

\author{
Mariana Cabral Schveitzer1, Vânia Marli Schubert Backes², Mônica Motta Lino³, Bruna Pedroso Canever", \\ Diana Coelho Gomes ${ }^{5}$
}

\footnotetext{
${ }^{1}$ Doutoranda em Enfermagem pela Escola de Enfermagem da Universidade de São Paulo. São Paulo, Brasil. E-mail: marycabral101@gmail.com

${ }^{2}$ Doutora em Enfermagem. Docente do Departamento de Enfermagem e Pós-Graduação em Enfermagem (PEN) da Universidade Federal de Santa Catarina (UFSC). Pesquisadora CNPq. Santa Catarina, Brasil. E-mail: oivania@ccs.ufsc.br

${ }^{3}$ Doutoranda em Enfermagem do PEN/UFSC. Bolsista Doutorado da CAPES. Santa Catarina, Brasil. E-mail: monicafloripa@ hotmail.com

${ }^{4}$ Mestranda em Enfermagem PEN/UFSC. Bolsista Mestrado/CNPq. Santa Catarina, Brasil. E-mail: olabruna@gmail.com

${ }^{5}$ Acadêmica de Enfermagem da UFSC. Bolsista de Iniciação Científica-PIBIC/CNPq. Santa Catarina, Brasil. E-mail: diana_ coelhoo@hotmail.com.com
}

\begin{abstract}
RESUMO: Este estudo tem o objetivo de identificar e caracterizar a produção científica dos grupos de pesquisa em educação em enfermagem das regiões Norte, Nordeste e Centro-Oeste do Brasil. Pesquisa documental, descritiva e exploratória, quantitativa. As regiões Norte, Nordeste e Centro-Oeste somam 98 grupos de pesquisa em enfermagem, 26\% do total nacional, sendo doze sobre Educação em Enfermagem. Existem registrados 140 pesquisadores, 120 estudantes e 22 técnicos. Entre os anos 1995-2008, estes grupos produziram 275 trabalhos completos em anais de eventos, 383 capítulos de livros, 135 livros e 455 artigos científicos. Formar e manter grupos de pesquisa em educação em enfermagem nos diversos cenários das regiões brasileiras requer disciplina e fomento das diversas organizações em educação e saúde para reduzir desigualdades e estimular a produção do conhecimento. Ademais, a produção científica pode ser uma das formas de socializar estes saberes e discutir a educação em enfermagem no espaço regional e nacional.
\end{abstract}

DESCRITORES: Enfermagem. Educação em enfermagem. Pesquisa em enfermagem.

\section{RESEARCH GROUPS IN NURSING EDUCATION: CHARACTERIZATION OF THREE REGIONS OF BRAZIL}

\begin{abstract}
This study aims to identify and characterize the scientific production from nursing education research groups in the North, Northeast, and Midwest regions of Brazil. It involves documental, descriptive, exploratory, and quantitative research. The North, Northeast, and Midwest regions account for 98 Nursing Research Groups, $26 \%$ of the national total, and twelve in the area of nursing education. There are 140 researchers, 120 students, and 22 technicians registered. From 1995 to 2008, these groups produced 275 research papers in conference proceedings, 383 book chapters, 135 books, and 455 scientific articles. Forming and maintaining nursing education research groups in the different scenarios of Brazil requires discipline and support from diverse health and education organizations to reduce inequalities and promote the production of knowledge. Therefore, scientific production may be one manner to socialize such knowledge and discuss nursing education on regional and national levels.
\end{abstract}

DESCRIPTORS: Nursing. Education, nursing. Nursing research.

\section{GRUPOS DE INVESTIGACIÓN EN ENFERMERÍA: CARACTERIZACIÓN DE TRES REGIONES DE BRASIL}

RESUMEN: Este estudio tiene como objetivo identificar y caracterizar la producción científica de los grupos de investigación en educación en enfermería de las regiones Norte, Nordeste y Centro-Oeste de Brasil. Estudio documental, descriptivo y exploratorio, cuantitativo. El Norte, Nordeste y Centro-Oeste tienen 98 grupos de investigación en enfermería, el 26\% del total nacional, y doce en educación en enfermería. Hay 140 investigadores, 120 estudiantes y 22 técnicos. Entre los años 1995-2008, estos grupos han producido 275 trabajos de investigación en las actas de congresos, 383 capítulos de libros, 135 libros y 455 artículos científicos. Construir y mantener grupos de investigación en la educación en enfermería en los diferentes escenarios necesita disciplina y la promoción de diversas organizaciones, de educación y salud, para reducir desigualdades y promover la producción del conocimiento. Además, la producción científica puede ser una forma de socializar y discutir Educación en Enfermería en los niveles regional y nacional.

DESCRIPTORES: Enfermería. Educación en enfermería. Investigación en enfermería. 


\section{INTRODUÇÃO}

O reconhecimento da complexidade da sociedade contemporânea e seu acelerado processo de modernização científica e tecnológica vem demandando novas formas de construção do conhecimento e, consequentemente, mudanças no processo de formação de profissionais competentes para o atendimento à saúde da população.

Essa necessidade de mudança decorre de elementos, tais como as novas modalidades de organização do mundo do trabalho em saúde e no mundo da escola (instituições formadoras), diante de exigências no perfil de profissionais, voltados para a produção do saber interdisciplinar.

$\mathrm{Na}$ área da enfermagem, a primeira metade do século XX destacou-se pela formação de recursos humanos e aspectos educacionais, já a segunda metade, pelo incentivo e incorporação da pesquisa no meio acadêmico, com a criação, primeiramente, no Brasil, dos cursos de mestrado (década de 1970) e doutorado (década de 1980), ${ }^{1}$ sendo que o objetivo primário da pós-graduação em enfermagem foi de qualificar docentes para o ensino superior. Posteriormente, a proposta enfatizou formar investigadores e, assim, gerar uma nova identidade da enfermagem, focada na produção de conhecimentos; identidade que, na época, não era reconhecida como própria da enfermagem. ${ }^{2}$

Juntamente com o aumento dos cursos de pós-graduação, observou-se o crescimento da produção científica em periódicos e em eventos específicos, mas não o impacto da produção científica da América Latina, quando comparada, em 1991, com os países da Europa e os Estados Unidos. Assim, como estratégia para atingir as exigências acadêmicas, ocorre, no Brasil, a partir da década de 1990, a constituição de grupos de pesquisa e núcleos de estudo em enfermagem. ${ }^{1,3}$

Em relação aos Grupos de Pesquisa em Educação em Enfermagem (GPEE), pesquisadores começaram a organizar-se a partir das orientações do Conselho Nacional de Desenvolvimento Científico e Tecnológico (CNPq), já a partir da década de 1980, no sentido de consolidar a sua base científica e a produção de conhecimentos em educação em enfermagem, ${ }^{4}$ visto que a participação nos grupos de pesquisa possibilita a indução de novos pesquisadores e constitui num diferencial na formação de docentes, discentes e profissionais da área, pois são espaços que permitem parcerias para o diálogo e que sejam realizadas intervenções e mudanças no cuidar e no ensinar em saúde.
No caso do Brasil, existe, atualmente, um crescimento desigual de grupos e institutos de pesquisa, os quais desenvolvem diferentes temáticas em nível de excelência, mas que devem estabelecer uma lista de prioridades para a pesquisa em enfermagem que variam conforme os interesses da população, do governo, da profissão ou da disciplina. ${ }^{1,3}$

Um exemplo dessa situação é a distribuição geográfica variada dos grupos de pesquisa no cenário brasileiro, que depende da localização dos cursos de pós-graduação, ${ }^{1}$ e assim, como há mais cursos de pós-graduação em enfermagem nas regiões Sul e Sudeste do Brasil, também, são nessas duas regiões que se concentram o maior número de grupos de pesquisa, quando comparados com o número de grupos das regiões Norte, Nordeste e Centro-Oeste. ${ }^{4}$

Diante desse contexto e considerando-se que para ocorrer avanços no processo formativo, é fundamental escrever e publicar os resultados das pesquisas, pois uma das formas de medir a produção do conhecimento é a partir da produção científica de um determinado setor acadêmico ${ }^{5}$, questiona-se: como se encontra a produção científica dos GPEE das regiões Norte Nordeste e Centro-Oeste do Brasil?

Para responder a essa pergunta, este estudo tem como objetivo identificar e caracterizar a produção científica dos GPEE das regiões Norte, Nordeste e Centro-Oeste do Brasil, registrados no CNPq, no censo de 2008.

\section{MÉTODO}

Trata-se de um estudo documental, exploratório-descritivo, quantitativo. Para a coleta dos dados, desenvolveram-se os seguintes passos: acesso ao site do CNPq; depois, em "Banco de Dados e Estatísticas"; após, em "grupos de pesquisa - Censos"; em seguida, em "Plano Tabular". A partir desse momento, foram selecionadas as seguintes variáveis: "Área de Atuação", "Por UF", "Por Instituição". Na sequência, foi realizado o filtro da primeira variável, sendo escolhida a área "Enfermagem". Assim, o sistema gerou uma tabela, que guiou toda a coleta dos dados, com o total de grupos de pesquisa em Enfermagem no Brasil, em 2008; discriminados a partir das variáveis selecionadas. Foram construídas tabelas no Microsoft Excel $2003^{\circledR}$, divididas por região geográfica do Brasil. 
Para as regiões Norte, Nordeste e Centro-Oeste constataram-se os seguintes elementos: instituições e o caráter institucional delas; número total de grupos de pesquisa na área de Enfermagem; número específico de GPEE, sendo que o critério para identificação foi apresentar a palavra "educação" ou sinônimos (ensino e formação) no nome do grupo; número total de Linhas de Pesquisa; presença de Programas de Pós-Graduação stricto sensu em Enfermagem; nome completo; ano de início e a sigla do Grupo de Pesquisa de Educação em Enfermagem; número, a formação, a titulação e a atuação profissional dos pesquisadores e técnicos dos GPEE; número, formação e a titulação dos estudantes dos GPEE; número de Bolsistas de Iniciação Científica entre os estudantes de graduação.

Os dados foram complementados por meio das informações disponibilizadas on-line no Diretório dos grupos de pesquisa do $\mathrm{CNPq}$, no Currículo Lattes dos pesquisadores, estudantes e técnicos e no site da Coordenação de Aperfeiçoamento de Pessoal de Nível Superior (CAPES) sobre os Programas de Pós-Graduação stricto sensu na Enfermagem.

Além da distribuição quantitativa da produção de artigos científicos, também foi verificada a qualificação dos periódicos onde esses têm sido publicados, por meio da lista de veículo de divulgação científica denominada Qualis/CAPES, referente ao Censo de 2008. Segundo esse indicador, a produção intelectual dos programas de pós-graduação strictu sensu é realizada pelas áreas de avaliação e passa por processo anual de atualização; esses veículos são enquadrados em estratos indicativos da qualidade A1, o mais elevado; A2, B1, B2, B3, B4, B5, C, com peso zero; e o aplicativo que permite a classificação e consulta ao Qualis das áreas, bem como a divulgação dos critérios utilizados para a classificação de periódicos é o WebQualis, disponível no portal virtual da CAPES.

Visto tratar-se de uma pesquisa documental, o estudo não foi submetido ao Comitê de Ética com Seres Humanos. Cabe, no entanto, ressaltar que foram seguidos os preceitos éticos contidos na resolução CNS 196/96, que trata da ética em pesquisas científicas.

\section{RESULTADOS}

Em acordo com o Censo/CNPq de 2008 há, no Brasil, 373 grupos de pesquisa em enferma- gem, 74 na região Sul, 201 na região Sudeste, 72 na região Nordeste, 19 na região Centro-Oeste e sete na região Norte. As regiões Norte, Nordeste e Centro-Oeste somam 98 grupos de pesquisa em enfermagem, ou seja, $26 \%$ do total nacional e, destes, 12 são GPEE, criados dois a partir da década de 90 e os outros dez a partir do ano 2000.

\section{Região Norte}

Na região Norte existe registrado um GPEE, identificado a partir da palavra "educação/ensino/formação" no nome do Grupo. Localizado no Estado do Pará, este grupo pertence a uma Instituição Pública de Ensino Superior, desde 2005. A região Norte do Brasil apresenta sete grupos de pesquisa em enfermagem. Constata-se, portanto, que $14 \%$ desses grupos trabalham com o tema "educação". Há 16 pesquisadores registrados nesse único GPEE. Destes, todos são da área de enfermagem. Há, também, seis estudantes de graduação em enfermagem, um bolsista de Iniciação Científica, e não há técnicos ligados ao GPEE. No tocante à formação do total de pesquisadores, foi encontrado apenas um título de pós-doutorado, um título de doutorado, 15 títulos de mestrado e dois de especialização na área de educação.

Entre os anos 1995-2008, o GPEE da região Norte do Brasil produziu 12 trabalhos completos em anais de eventos, 15 capítulos de livros, três livros e 18 artigos científicos. Visto que a partir do ano 2000 foram publicados 93,75\% de toda a produção do GPEE da região Norte do Brasil, especialmente, os últimos dois anos (2007-2008) correspondem a 38,88\% da publicação total de artigos científicos.

No período entre 2004-2008 foram publicados 11 artigos científicos, o que indica um índice de produtividade científica na proporção de menos de um artigo por pesquisador. Dessa forma, para que a análise desse resultado não seja restrita ao volume produzido; optou-se, neste trabalho, por verificar a qualificação dos periódicos que têm sido publicados, por meio da lista de veículo de divulgação científica denominada Qualis/CAPES, estes dados estão descritos na Tabela 1.

Dessa forma, a região Norte apresentou, entre o total de 11 artigos produzidos nesse período, oito que foram publicados em revistas classificadas pelo Qualis/CAPES de acordo com o censo 2008 em conceitos A1, A2, B1 e B2, ou seja, 72\% da produção científica. 
Tabela 1 - Distribuição da produção de artigos científicos do grupo de pesquisa em educação em enfermagem da região Norte do Brasil, no período da coleta (2004-2008), segundo Qualis/ CAPES para a área de Enfermagem. CAPES, 2009

\begin{tabular}{lc}
\hline Qualis/CAPES & $\mathbf{2 0 0 4} \mathbf{- 2 0 0 8}$ \\
\hline A1 & 1 \\
A2 & 2 \\
B1 & 2 \\
B2 & 3 \\
B3, B4, B5 & 2 \\
C & - \\
Não consta* & 1 \\
\hline Total & 11 \\
\hline
\end{tabular}

* Não consta na lista Qualis/CAPES.

\section{Região Nordeste}

A região Nordeste do Brasil apresenta 72 grupos de pesquisa em enfermagem, distribuídos em 21 Instituições de Ensino Superior (IES) públicas e privadas. Destes, sete apresentam-se como GPEE, sendo que um grupo pertence ao estado da Bahia, um ao estado do Ceará, um ao Maranhão, dois ao estado do Piauí e dois ao estado do Rio Grande do Norte, todos distribuídos em seis instituições públicas. Levando-se em conta o número total de grupos de pesquisa em Enfermagem na região Nordeste do Brasil, constata-se que 27\% destes trabalham com o tema "educação".

Existem cadastrados nos GPEE 69 pesquisadores. Desse montante, 67 de enfermagem e dois de outras áreas do conhecimento, a saber, educação física e odontologia. A titulação dos pesquisadores distribui-se da seguinte forma: dois possuem pós-doutorado, 24 doutorado, 25 mestrado, e 10 especialização. Cabe ressaltar que desse total, 25 possuem titulações (especialização, mestrado ou doutorado) na área da educação.

Ainda, esses GPEE contam com 97 estudantes, sendo oito bolsistas de iniciação científica. Os técnicos totalizam nove, entre eles, oito possuem formação em enfermagem e um não informa. Sobre a titulação: dois são mestres, dois especialistas e cinco graduados. Entre os técnicos, dois trabalham na docência, dois na assistência, um em ambos, quatro não informaram.

Esse contingente está distribuído da seguinte maneira: na Bahia são quatro pesquisadores, 15 estudantes e sete técnicos; no Ceará são 17 pesquisadores, 42 estudantes e nenhum técnico; no Maranhão são cinco pesquisadores, dez estudantes e nenhum técnico; no Piauí, são 11 pesquisadores, um estudante e nenhum técnico; e, no Rio Grande do Norte, são 32 pesquisadores, 29 estudantes e um técnico.

No tocante produção dos GPEE, entre os anos 1995-2008, a região Nordeste do Brasil produziu o total de 126 trabalhos completos em anais de eventos, 255 capítulos de livros, 45 livros e 278 artigos científicos. A partir do montante das produções citadas acima dos GPEE da região Nordeste, seguem descritos na tabela 2 os resultados que dizem respeito aos artigos científicos.

Tabela 2 - Distribuição temporal (2004-2008) da produção de artigos científicos dos grupos de pesquisa em educação em enfermagem da região Nordeste do Brasil. CNPq, 2009

\begin{tabular}{cccccc}
\hline Ano & BA & CE & MA & PI & RN \\
\hline 2004 & 4 & 29 & 3 & 1 & 6 \\
2005 & 4 & 36 & 4 & 5 & 4 \\
2006 & 4 & 22 & 4 & 2 & 3 \\
2007 & 4 & 38 & 9 & 16 & 6 \\
2008 & 4 & 40 & - & 19 & 11 \\
\hline Total & $\mathbf{2 0}$ & $\mathbf{1 6 5}$ & $\mathbf{2 0}$ & $\mathbf{4 3}$ & $\mathbf{3 0}$ \\
\hline
\end{tabular}

Esse resultado infere que a média produtiva de um pesquisador foi de quatro artigos nos últimos cinco anos. A distribuição dessa média, no entanto, muda, consideravelmente, a partir de sua percepção por Estado, nos últimos cinco anos, o índice produção versus pesquisador foi de cinco artigos na Bahia, de nove a 10 artigos no estado do Ceará, quatro artigos no estado do Maranhão, de três a quatro artigos no Piauí e menos de um artigo por pesquisador no Rio Grande do Norte.

Além da distribuição quantitativa da produção de artigos científicos; também, foi verificada a qualificação dos periódicos os quais têm sido publicados, por meio da lista de veículo de divulgação científica denominada Qualis/CAPES, conforme apresentado na tabela 3.

Entre os artigos publicados pelos GPEE da região Nordeste constata-se que, do total de 278 artigos, $66 \%$ foram publicados em revistas classificadas pelo Qualis/CAPES, de acordo com o censo 2008 em conceitos A1, A2, B1 e B2. 
Tabela 3 - Distribuição da produção de artigos científicos dos grupos de pesquisa em educação em enfermagem da região Nordeste do Brasil, por ano (2004-2008), segundo Qualis/CAPES para a área de enfermagem. CAPES, 2009

\begin{tabular}{lccccc}
\hline Qualis/CAPES & BA & CE & MA & PI & RN \\
\hline A1 & - & 2 & - & 1 & 1 \\
A2 & 6 & 14 & 1 & - & - \\
B1 & 7 & 51 & 2 & 21 & 4 \\
B2 & 1 & 63 & - & 7 & 4 \\
B3, B4, B5 & 6 & 31 & 6 & 6 & 11 \\
C & - & 1 & 1 & 2 & - \\
Não consta* & - & 3 & 10 & 6 & 10 \\
\hline Total & $\mathbf{2 0}$ & $\mathbf{1 6 5}$ & $\mathbf{2 0}$ & $\mathbf{4 3}$ & $\mathbf{3 0}$ \\
\hline * Não consta na lista Qualis/CAPES. & & &
\end{tabular}

\section{Região Centro-Oeste}

Na região Centro-Oeste do Brasil existe o cadastro de quatro GPEE, identificados a partir da palavra "educação/ensino/formação", no nome do grupo. Destes, dois são pertencentes ao Distrito Federal, um ao Mato Grosso do Sul e um ao Mato Grosso, distribuídos em três instituições públicas. Levando-se em conta que o número total de grupos de pesquisa em enfermagem na região Centro-Oeste do Brasil é 19, constata-se que 21\% destes trabalham com o tema educação.

Há 55 pesquisadores registrados nos GPEE, sendo 34 no Distrito Federal, 11 no Mato Grosso do Sul e dez no Mato Grosso. Deste total, 40 são da área de enfermagem e 12 são de outras áreas do conhecimento como: Ciências sociais, medicina, psicologia, fisioterapia, administração, matemática, e três não especificados. No tocante à formação, apenas dois possuem pós-doutorado, 33 doutorado, 22 mestrado e um especialização, sendo um total de 17 que apresentam titulações (especialista, ou mestre ou doutor) na área da educação.

Ainda, estão registrados,17 estudantes, sendo nenhum bolsista e 13 técnicos no Distrito Federal, quatro estudantes e dois bolsistas PIBIC e nenhum técnico no Mato Grosso, sendo que não existem estudantes ou técnicos registrados no GPEE do Mato Grosso do Sul.

Os técnicos totalizam 13 no Distrito Federal. Entre eles, seis possuem formação em enfermagem, um em outra área de conhecimento, história, e seis não informaram. Sobre a titulação, 11 são especialistas e dois graduados. Do total de técnicos, três trabalham com assistência, três em docência e assistência e sete não informaram.
Entre os anos 1995-2008, os GPEE da região Centro-Oeste do Brasil produziram 173 trabalhos completos em anais de eventos, 113 capítulos de livros, 87 livros e 159 artigos científicos.

A partir desse total de produções dos GPEE da região Centro-Oeste do Brasil, optou-se em apresentar, com maiores detalhes, na tabela 4, os resultados referentes aos artigos científicos, visto sua relevância específica para o setor de educação em enfermagem e saúde.

Tabela 4 - Distribuição temporal (2004-2008) da produção de artigos científicos dos grupos de pesquisa em educação em enfermagem da região Centro-Oeste do Brasil. CNPq, 2009

\begin{tabular}{lccc}
\hline Ano & DF & MS & MT \\
\hline $\mathbf{2 0 0 4}$ & 21 & 1 & 7 \\
$\mathbf{2 0 0 5}$ & 22 & 2 & 2 \\
$\mathbf{2 0 0 6}$ & 21 & 9 & 9 \\
$\mathbf{2 0 0 7}$ & 25 & 2 & 4 \\
$\mathbf{2 0 0 8}$ & 25 & 6 & 3 \\
\hline Total & $\mathbf{1 1 4}$ & $\mathbf{2 0}$ & $\mathbf{2 5}$ \\
\hline
\end{tabular}

Nos últimos cinco anos (2004-2008), os GPEEs da região Centro-Oeste do Brasil registraram a publicação do montante de 159 artigos científicos. Destes, 114 foram produzidos pelos GPEEs de Distrito Federal, 20 pelo grupo do Mato Grosso do Sul e 25 pelo grupo do Mato Grosso. Esse resultado infere que a média produtiva de um pesquisador foi de dois a três artigos nos últimos quatro anos. A distribuição dessa média, no entanto, muda, consideravelmente, a partir de sua percepção por Estado. Nos últimos cinco anos, o índice de produção versus pesquisador foi de três a quatro no Distrito Federal, de um a dois artigos no Mato Grosso do Sul e de dois a três artigos no Mato Grosso.

Além da distribuição quantitativa da produção de artigos científicos, também foi verificada a qualificação dos periódicos que têm sido publicados, por meio da lista de veículo de divulgação científica denominada Qualis/CAPES, conforme descrito na Tabela 5.

Assim, constata-se que não há registro de artigo científico publicado em revista classificada pelo Qualis/CAPES em conceito A1 pelos GPEEs da região Centro-Oeste e que $54 \%$ do total da produção científica foi publicada em revistas classificadas em conceitos B3, B4, B5, C ou que não constam na lista Qualis/CAPES de acordo com o censo 2008. 
Tabela 5 - Distribuição da produção de artigos científicos dos grupos de pesquisa em educação em enfermagem da região Centro-Oeste do Brasil, por ano (2004-2008), segundo Qualis/ CAPES para a área de Enfermagem. CAPES, 2009

\begin{tabular}{lccc}
\hline Qualis/CAPES & MS & DF & MT \\
\hline A1 & - & - & - \\
A2 & 2 & 7 & 1 \\
B1 & 5 & 26 & 14 \\
B2 & - & 16 & 3 \\
B3, B4, B5 & 12 & 46 & 3 \\
C & - & 2 & - \\
Não consta* & 1 & 17 & 4 \\
\hline Total & $\mathbf{2 0}$ & $\mathbf{1 1 4}$ & $\mathbf{2 5}$ \\
\hline
\end{tabular}

* Não consta na lista Qualis/CAPES.

\section{DISCUSSÃO}

A pesquisa, na área de educação em enfermagem no Brasil, tem avançado muito, nos últimos anos, com a criação e a consolidação dos grupos de pesquisa, na última década. Nota-se que, em relação ao número absoluto dos cursos de graduação em enfermagem, o maior contingente de GPEEs encontra-se nas IES Públicas. ${ }^{4}$ Esse avanço vem ao encontro das tendências da pós-graduação stricto sensu em enfermagem, que desempenha papel fundamental enquanto estimuladora do desenvolvimento científico, sendo que todos os GPEEs das Regiões Norte, Nordeste e Centro-Oeste encontram-se, exclusivamente, nas IES Públicas.

Através da coleta e análise de dados sobre a região Norte, pode-se evidenciar a grande necessidade de investimentos na qualificação de pesquisadores em enfermagem da região; bem como a ampliação e fortalecimento de grupos de pesquisa em enfermagem e GPEE, pois o único GPEE existente, ainda é frágil em relação ao número de pesquisadores doutores, estudantes e técnicos, a interdisciplinaridade e a integração docente-assistencial. Isso corrobora com a política de formação de pesquisadores para a região Norte, incentivada pela CAPES, por meio dos Doutorados Interinstitucionais; pois, assim, foi possível estruturar o primeiro programa de pós-graduação stricto sensu para desenvolver e consolidar a enfermagem científica na região, com o desenvolvimento do DINTER Enfermagem, entre a Universidade Federal de Santa Catarina e a Universidade Federal do Pará.
Em relação aos resultados referentes à região Nordeste, o fato de $27 \%$ dos grupos da região tratarem do tema educação é bastante promissor, todavia, a maioria possui formação recente (a partir do ano 2000) e, para manter e ampliar os GPEEs, é fundamental investir na qualificação dos pesquisadores, na ampliação do número de bolsistas de pesquisa em relação ao total de estudantes, e no número de técnicos que participam dos grupos, no sentido de diminuir a distância dos ambientes de trabalho e do ensino. Ademais, eventos para discutir e apresentar os trabalhos dos GPEEs podem ser interessantes para realizar a troca de informações e apoio entre pesquisas de caráter regional, como por exemplo, os encontros que estimularam a criação dos Seminários Nacionais de Diretrizes para Educação em Enfermagem (SENADEN) ${ }^{6}$

Quanto aos resultados da região Centro-Oeste, interessante diagnosticar que dois estados ainda não possuem GPEE, como é o caso de Tocantins e Goiás, para que $100 \%$ da região esteja coberta, como é o caso da região Sul. ${ }^{7}$ Existe, também, mais de um GPEE na mesma universidade pública, demonstrando a infinidade do tema educação e as diferentes possiblidades de abordagem e pesquisa. Além do mais, as evidências encontradas indicam a necessidade de se investir no processo da integração ensino-serviço, com a participação de mais técnicos e, também, de estudantes; ressaltando que a participação de alunos de graduação nos grupos de pesquisa, favorece a indução de formação do perfil de pesquisador na enfermagem. ${ }^{4}$

A partir da necessidade de divulgar o conhecimento produzido por esses pesquisadores em enfermagem, multiplicaram-se o número de artigos científicos publicados em revistas nacionais e internacionais. Também incentivado e exigido, pelos programas de pós-graduação e órgãos de fomento aos professores e alunos, como critério para avançar com os investimentos e os conceitos avaliativos dos programas de pós-graduação, visto a realidade atual de publicar ou perecer. ${ }^{8,9}$

Dessa forma, esse vasto campo de informação criou condições para buscar a qualidade do material impresso e, atualmente, disponível nas plataformas digitais. Situação essa que, também, divide pesquisadores; de um lado ficam as revistas que têm que sobreviver no mercado capitalista de inscrição e referência e, do outro, os pesquisadores e profissionais que buscam nos artigos disponíveis fontes de informação para guiar a prática de cuidado e para tal tendem a escolher, normalmente, aqueles disponíveis on-line, gratuitamente. ${ }^{10}$ 
Nesse sentido, vale destacar os resultados sobre a classificação Qualis/CAPES dos artigos do GPEE da região Norte, pois a maioria (70\%) encontrava-se publicada em revistas conceito A e $B$, e que, por estarem indexadas em diferentes bases de dados virtuais e gratuitos, permitem a sua ampla distribuição e acesso, por diferentes profissionais, de outras regiões brasileiras.

Todavia, essa realidade muda quando se analisa os artigos da região Nordeste e Centro-Oeste, ainda que seja expressivo o número de artigos publicados em revistas A e B (em torno de 70 artigos por região nos últimos cinco anos). Mais de $50 \%$ dos trabalhos foram publicados em revistas B3, B4, B5, C ou em revistas que não possuem classificação pelo Qualis/CAPES, que dificultam a circulação e a leitura dos conteúdos publicados.

Dessa forma, percebe-se a importância dos programas de pós-graduação e do CNPq na criação e consolidação dos GPEEs; inclusive com a atual necessidade de ampliar os investimentos e os processos de avaliação dos programas e, por consequência, dos grupos de pesquisa e seus membros, em caráter multidisciplinar, na busca de uma maior visibilidade à enfermagem. ${ }^{1,4}$

\section{CONSIDERAÇÕES FINAIS}

Esse estudo procurou sistematizar e criar um banco de dados da produção científica dos GPEEs nas Regiões Norte, Nordeste e Centro-Oeste do Brasil e determinar as fontes de divulgação dessas produções científicas, visto que o processo de construção do conhecimento é uma prática social desafiadora, que varia conforme as regiões brasileiras, e depende de recursos humanos compromissados com o saber da enfermagem.

Formar e manter GPEEs, nos diversos cenários das regiões brasileiras, requer disciplina e fomento das diversas organizações em educação e saúde para reduzir desigualdades e estimular a produção do conhecimento; não somente dentro das universidades, mas também nos ambientes de trabalho em saúde.

Nessa perspectiva, os artigos científicos podem ser reconhecidos como uma das formas de socializar esses saberes e de discutir a educação em enfermagem no espaço regional e nacional. Os grupos de pesquisa, que contam com a participação de alunos, técnicos e pesquisadores de diferentes áreas, trabalhando e pensando em conjunto, podem ampliar as possibilidades para aproximar o mundo da escola e do trabalho e diminuir iniquidades no processo de ensinar e cuidar.

\section{AGRADECIMENTOS}

Ao CNPq pelo apoio financeiro e ao Grupo de Pesquisas em Educação em Enfermagem e Saúde - EDEN/UFSC, exemplo concreto de construção coletiva do saber, aprender e ensinar.

\section{REFERENNCIAS}

1. Barbosa SFF, Sasso GTM, Berns I. Enfermagem e tecnologia: análise dos grupos de pesquisa cadastrados na Plataforma Lattes do CNPQ. Texto Contexto Enferm. 2009 Jul-Set; 18(3):443-8.

2. Jiménez MA, Serrano CG, Mendoza EV, Olaya CM. Contexto de la producción investigativa en educación em enfermería en Colombia 1995-2004. Av Enferm. 2005 Ene-Jun; 23(1):5-17.

3. Carvalho EC. A produção do conhecimento em enfermagem. Rev Latino-am Enfermagem. 1998 Jan; 6(1):119-22.

4. Backes VMS, Canever BP, Ferraz F, Lino MM, Prado ML, Reibnitz KS. grupos de pesquisa em educação em enfermagem da região Sul do Brasil. Rev Gaúcha Enferm. 2009 Jun; 30(2):249-56.

5. Morse JM, Field PA. Qualitative research methods for health professionals. $2^{\mathrm{a}}$ ed. London (UK): Sage Publications; 1985.

6. Moura A, Liberalino FN, Silva FV, Germano RM, Timóteo RPS. SENADEn: expressão política da educação em enfermagem. Rev Bras Enferm 2006; 59(Esp):441-53.

7. Lino, MM. Produção científica dos grupos de enfermagem da região Sul do Brasil [dissertação]. Florianópolis (SC): Universidade Federal de Santa Catarina, Programa de Pós-Graduação em Enfermagem; 2009.

8. Narvai PC. Pós-graduação, sistema Qualis e futebol. Rev Adusp. 2009 Out; 45: 51-6.

9. Logan, W. The academic man: a study in the sociology of a profession. New Brunswick (NJ): Transaction Publishers; 1995.

10. Greenhalg T. Artigos que vão além dos números (pesquisa qualitativa). In: Greenhalg T. Como ler artigos científicos: fundamentos da medicina baseada em evidências. Porto Alegre (RS): Artmed; 2008. p.187-200. 